9. Oxalatkristalle sind fast Kristalldrusen vom Mesophyll-Typus ................... Folium Stramonii

10. Oxalatkristalle sind Gemische von P'rismen und Sphäriten vom Mesophyll-Typus......... Folium Hyoscyami

2. Die Epidermis besteht aus polygonalen Zellen

11. Haben Haare

13. Einzellige Haare sind meist nicht gekrümmt. Im Mesophyll sind relativ viel Oxalatdrusen . . . . Folium Sennae Tinnevelly

14. Einzellige Haare sind meist gekrümmt, im Mesophyll sind relativ wenig Oxalatdrusen ..... Folium Sennae Alexandrinae

12. Keine Haare oder man sieht sie kaum

15. Im Mesophyll sind Oelräume ... . . Folium Eucalypti

16. Im Mesophyll sind keine Oelräume

17. Schwammparenchym besteht aus unregelmässigen, verzweigten Zellen; im Mesophyll kommen viele Oxalatdrusen vor .................... Folium Pruni macrophyllae

18. Schwammparenchym besteht aus runden bezw. elliptischen Zellen, die Oxalatkristalle sind hauptsächlich Einzelkristalle vom Nerven-Typus ....... Folium Uvae Ursi.

\title{
Über die Konstitution des Forsythin (II. Mitteil.)
}

von

Senkichi Kunimine und Shintaro WadA

[Aus der Pharmazeutischen Fachschule zu Keijo.]

(Eingegangen am 5. Juli 1938.)

Verfasser haben in ihrer ersten Mitteilung1) für Forsythin die Konstitutionsformel I bezw. II vorgeschlagen. Durch Nitrierung von $d$-Forsythigenol-methyläther (V), der durch Methylierung des Aglykons vom Forsythin erhalten worden ist, entstanden 
Dinitro- $d$-Forsythigenol-methyläther (Fp. $180^{\circ},[\alpha]_{D}^{23}=+47.4^{\circ}$ ) und 4-Nitroveratrol. Behandelt man Dinitro- $d$-Forsythigenol-methyläther mit Salpetersäure, so entsteht 4,5-Dinitroveratrol, so dass die Nitrogruppe wie in der Formel VII gebunden ist.

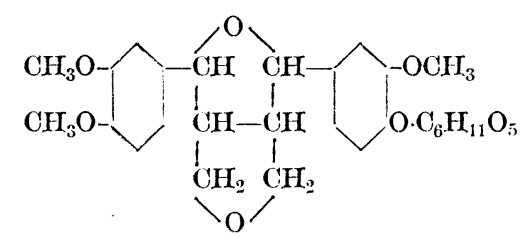

(I)

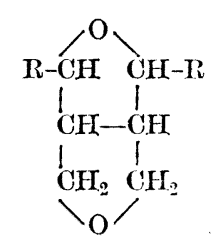

(III)

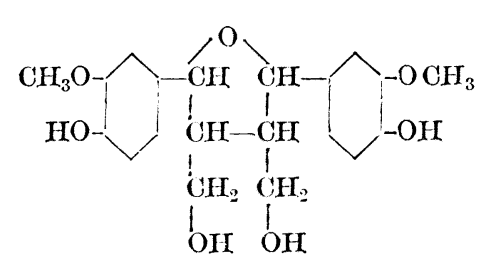

(VIII)

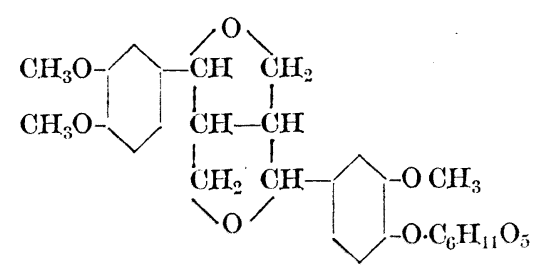

(II)
(IV)

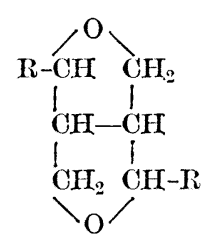

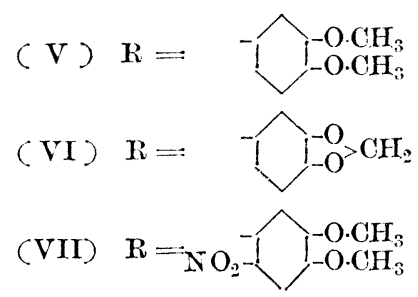<smiles>COCC1C(O)C(CO)C(CO)C(C2CCC(O)C(O)C2)C1CCO</smiles>

Von Substanzen, welche kondensierter Tetrahydrofuranting aufweisen und gleichzeitig Veratrolgruppen haben, existieren Pinoresinoldimcthyläther'2) (V), Eudesmin ${ }^{3)}$ (V) und Asarinin ${ }^{4}$ (VI), Sesamin ${ }^{5}$ (VI) gehören zu Substanzen mit kondensiertem Tetrahydrofuranring mit Piperonylgruppen. Kaku und seine Mitarbeiter haben $l$ Asarinin, $d$-Sesamin nach Aufspaltung der Methylendioxygruppe in Tetramethyläther übergeführt und Eudesmin, Pinoresinoldimethyläther erhalten, gleichzeitig aber auch Substanzen, welche bei $133-134^{\circ}$ schmelzen und haben letztere Epi-Eudesmin, EpiPinoresinoldimethyläther ${ }^{-6)}$ genannt.

Da für unseren $d$-Forsythigenol-methyläther die Konstitutionsformeln III bezw. IV (mit $\mathrm{R}=\mathrm{CH}_{3} \mathrm{CH}_{3} \mathrm{O}-$ ) vorgeschlagen wurden, ferner die Formeln III bezw. IV dem Pinoresinoldimethyläther, Eudesmin zugeschrieben werden, so sollten beide dasselbe 
Kohlenstoffgerüst aufweisen und die beiden Veratrolgruppen sollten am gleichen Kohlenstoff gebunden sein. Um dies zu beweisen, wurde $d$-Forsythigenolmethyläther mit Chlorwasserstoff im Methanol erhitzt, wodurch die Substanz A (Fp. 107-108 $[a]_{\mathrm{D}}^{150}=+64.2^{\circ}$, die unveränderte Substanz $d$-Forsythigenol-methyläther $\mathrm{B}$ und die Substanz C, welche höheren Schmelzpunkt und höheres spezifisches Drehungsvermögen als das Ausgangsmaterial zeigt, erhalten wurden.

A enthält kein Chlor. Ihm fehlt die Hydroxylgruppe und durch Oxydation mit Kaliumpermanganat entsteht nur Veratrumsäure. Durch Chlorwasserstoffsäure in Methanol bildet sich $d$-Forsythigenolmethyläther, so dass A kein Phenylnaphthalinderivat ist wie Olivil7) (VIII), aus welchem sich Isoolivil (IX) bildet oder wie Lariciresinol ${ }^{8)}$, woraus Isolariciresinol entsteht. Farbenreaktion, Schmelzpunkt, spezifisches Drehungsvermögen und alle Konstanten der Dinitroverbindung stimmen mit denen des Pinoresinoldimethyläthers überein. Mithin sind ganz bestimmt $d$-Forsythigenolmethyläther und Pinoresinoldimethyläther Diastereoisomere. C lässt sich schwer reinigen; eine Substanz, welche bei $133-134^{\circ}$ schmilzt, wurde zwar erhalten, aber sie enthält noch $d$-Porsythigenolmethyläther. Sie wurde über die Nitroverbindung gereinigt. Verfasser bekamen eine Dinitroverbindung vom Fp. $230^{\circ}$ und $[\alpha]_{\mathrm{D}}^{130}=+119.4^{\circ}$. Auf Grund folgender Tatsachen, dass

1) C kein Phenylnaphtalinderivat ist, dass

2) eine Dinitroverbindung erhalten wurde, dass

3) Hydroxylgruppen fehlen

und aus sonstigen chemischen Eigenschaften haben sie $\mathrm{C}$ als Diastereoisomer des $d$-Forsythigenolmethyläthers aufgefasst und $d$-Epiforsythigenolmethyläther genannt.

Auch haben Verfasser die Methylendioxygruppe des $d$-Sesamins aufgespalten und in Tetramethyläther übergeführt und die Verbindung A (Fp. 107-108 $)$, B (Fp. 128$128^{\circ} .5$ ) und die Substanz C, deren Fp. und [a], hoeher liegen, erhalten. A entspricht dem Pinoresinoldimethyläther und bei der Mischprobe mit Pinoresinoldimethyläther aus $d$-Forsythigenolmethyläther tritt keine Depression ein. $\mathrm{B}$ ist $d$-Forsythigenolmethyläther und $\mathrm{C}$ entspricht dem Epiforsythigenolmethyläther.

$l$-Forsythigenolmethyläther und $l$-Epiforsythigenolmethyläther sind zwar noch nicht untersucht worden, aber da sie Antipoden von $d$-Forsythigenolmethyläther, $d$-Epiforsythigenolmethyläther sind, so verstehen sich alle Konstanten von selbst und wenn 
man die Methylendioxygruppe von $l$-Sesamin, $l$-Asarinin aufspaltet und in Tetramethyläther überführt, wird man sie erhalten.

\title{
Literatur
}

1) Diese Zeitschrift 58, $\unlhd 5$ (1938),

2) und 3) Annual reports on the progress of chemistry 1936, $276 ; \mathrm{T}$. Kaku und H. Ri: Diese Zeitschrift, 57, $2 s 9$,

4) und 5) T. Kaku und న. Kutani: Biochem. Z. 201, 454-463 (192S); T. Kaku und H. Ri,: Diese Zeitschrift 56, 80 (1936); 57, 184, 289 (1937); Huang Minlon, B. 70, 951 (1937),

6) Diese Zeitschrift 57, 289 (1937),

7) M. 52, $163(1929$,

8) Soc. 1937, ist.

\section{Ein Bestandteil der Rinde von Fraximus Sieboldiana Blume (Oleaceae).}

\author{
VoI \\ Haruya Shimada \\ [Aus der Pharmazeutischen Fachschule zu Kyoto.]
}

(Eingegangen am 23. Juni 1938.)

„Koba no Toneriko" (Syn. „Aodamo“) d. i. Fraxinus Sieboldiana Blume (F. Sieboldiana Bl. var. serrata Nakai) ist eine zur Gattung Fraxinus gehoerige, in den Gegenden von Honshu (Hauptinsel Japans) und Kyushu sehr verbreitet vorkommende Pflanze. "Toneriko" (Fraxinus Japonica Blume), welche mit derselben nahe verwandt ist und seit alters her als die chinesische Droge ts'in p'i angegeben wird, wird für Augenerkrankungen viel gebraucht. In der Gegend von Kyoto kommt Eraxinus Sieboldiana Bl. wild und besonders häufig vor, während Fraxinus Japonica Blume eher seltener vorkommt. Man muss vermuten, dass die seit alters gebrauchte ts'in $\mathrm{p}^{\prime} \mathbf{i}$ nicht allein der gegenwärtigen japanischen Pflanze Fraxinus Japonica Bl. entspricht, sondern, dass mann dafür alle Varietäten der Gattung Fraxinus verwendet hat. 